\title{
Habitat structure: A fundamental concept and framework for urban soil ecology
}

\section{Loren B. Byrne}

(C) Springer Science + Business Media, LLC 2009

Habitat structure is defined as the composition and arrangement of physical matter at a location. Although habitat structure is the physical template underlying ecological patterns and processes, the concept is relatively unappreciated and underdeveloped in ecology. However, it provides a fundamental concept for urban ecology because human activities in urban ecosystems are often targeted toward management of habitat structure. In addition, the concept emphasizes the fine-scale, on-the-ground perspective needed in the study of urban soil ecology. To illustrate this, urban soil ecology research is summarized from the perspective of habitat structure effects. Among the key conclusions emerging from the literature review are: (1) habitat structure provides a unifying theme for multivariate research about urban soil ecology; (2) heterogeneous urban habitat structures influence soil ecological variables in different ways; (3) more research is needed to understand relationships among sociological variables, habitat structure patterns and urban soil ecology. To stimulate urban soil ecology research, a conceptual framework is presented to show the direct and indirect relationships among habitat structure and ecological variables. Because habitat structure serves as a physical link between sociocultural and ecological systems, it can be used as a focus for interdisciplinary and applied research (e.g., pest management) about the multiple, interactive effects of urbanization on the ecology of soils.

Note: The paper was intended for inclusion in this special feature on soils but was accidentally published separately. The abstract is from Urban Ecosyst (2007) 10:225-274. 